\title{
Authoring Systems Delivering Reusable Learning Objects
}

doi:10.3991/ijet.v4s2.812

\author{
J. Schreurs ${ }^{1}$, R. Dalle ${ }^{2}$, George Sammour ${ }^{1}$ \\ ${ }^{1}$ Hassselt University, Diepenbeek, Belgium \\ ${ }^{2}$ EduWEST.be, Ypres, Belgium
}

\begin{abstract}
A three layer e-learning course development model has been defined based on a conceptual model of learning content object. It starts by decomposing the learning content into small chunks which are initially placed in a hierarchic structure of units and blocks. The raw content components, being the atomic learning objects (ALO), were linked to the blocks and are structured in the database. We set forward a dynamic generation of LO's using re-usable e-learning raw materials or ALO's
\end{abstract}

In that view we need a LO authoring/ assembling system fitting the requirements of interoperability and reusability and starting from selecting the raw learning content from the learning materials content database. In practice authoring systems are used to develop e-learning courses. The company EDUWEST has developed an authoring system that is database based and will be SCORM compliant in the near future.

Index Terms-Learning Object, authoring system, reusability of learning content, interoperability of e-learning module

\section{INTRODUCTION}

In an e-learning situation the source learning content has to be converted into e-learning content, being learning objects (LO)

The source learning content has been decomposed first in a set of chapters. Each chapter will be converted into an independent e-learning module. In this chapter we can identify one or more units of content.

That content of a unit will be decomposed manually into small chunks of content or blocks, sub-blocks. The content corresponding to the blocks and sub-blocks have to be decomposed in raw content components or atomic learning objects (ALO) (text, figures, summary of text, keywords of text) and have to be supplemented with additional ALOs to create interactivity and animations.

LO1: CH1: Introduction

LO2: CH2: Supply of ICT professionals

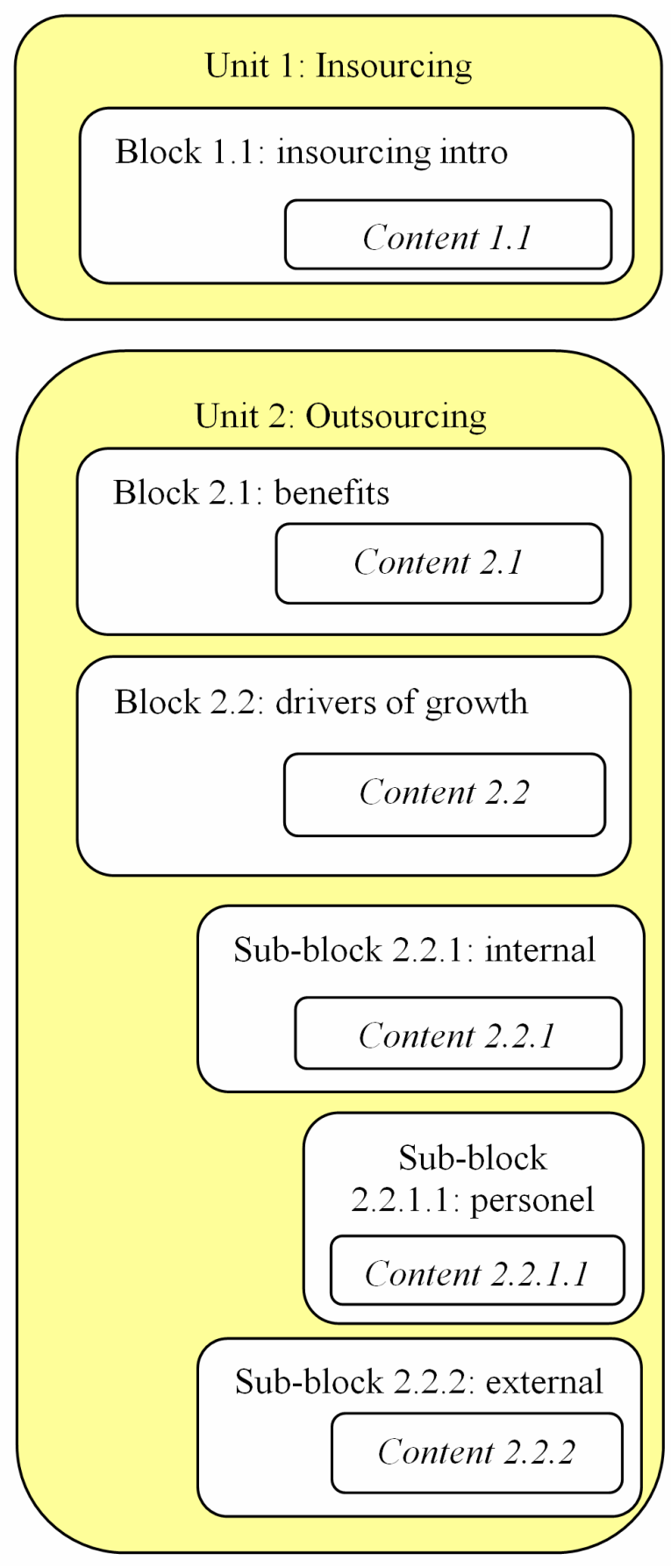

Figure 1. tree-structured source content 
The LO's representing a course module, corresponding to a chapter content, will include the tree structured learning content presented as a set of pages in a predefined layout realised as a scenario composition of the ALOs.

A course can be built by packaging learning objects or the course modules. The requirements of interoperability (content from multiple sources working equally well with different learning systems) and of reusability (content developed in one course being transferable to another context) have to be fulfilled.

\section{The Process of CONVERTING CONTENT to ReUSABLE LEARNING OBJECTS.}

\section{A. The decomposition of the learning content}

The e-learning project development team consists of a content owner/ expert, an instructional designer and a programmer-developer. The instructional designer is decomposing the original content and creates the treestructure of the chunks of content.

The decomposition of the learning content is a manual process. The source learning content has to be decomposed and structured as a hierarchical tree of chapters, units and blocks. [10-6]. (figure 1) A course is similar with a book while a chapter contains many blocks, being smaller content parts. If the chapter can really be split into more individual content topics, or which can be learned as individual topics, then the learning content is split into two or more independent content units, put together in a sequence. The learner will subsequently learn them afterwards as one logical content module. Each unit is composed of a set of blocks, corresponding to smaller content parts, being structured as a relational tree.

In a second part of the decomposition phase, that block content is split in raw content elements or ALOs: the full text, its summary, figures and tables, and the key terms of its content. Some other ALOs can be added, such as a video telling the story, Q \& A, URL of additional information, etc. Accordingly, metadata will be defined for all content components [9].

\section{B. The learning content management system (LCMS)}

The LCMS is responsible for the production, reuse, the management and the storage of the learning content. It is composed of the following functions [16]

1) authoring system

2) content delivery interface

3) administrative management systems

4) storage of the content in a database;

In the LCMS learning content will be created and uploaded in the database, and LO's will be created and also stored in the DB.

Starting from the source learning content, the hierarchical structure of units and blocks are identified in it. To create the learning objects the content will be structured in the database following the hierarchical content structure. And to assemble the content linked with the blocks the content components or ALOs are tracked from the data tables. ALOs include full and summary text, figures, questions and answers, audio files.

\section{Requirements of re-usability of learning content and} of interoperability of learning objects.

For re-usability, we have the following possibilities in mind: first, based on the same source content, we can make a selection of blocks we bring in the LO, second we bring more LOs with the same content, but presented in another layout fitting the preference of the user, and fitting the used appliance of the learner.

In our previous paper on the subject (15) we explained the re-usability and the operability of LO's and introduced the SCORM standard. In figure(2) re-usability and interoperability has been positioned in the development life cycle.

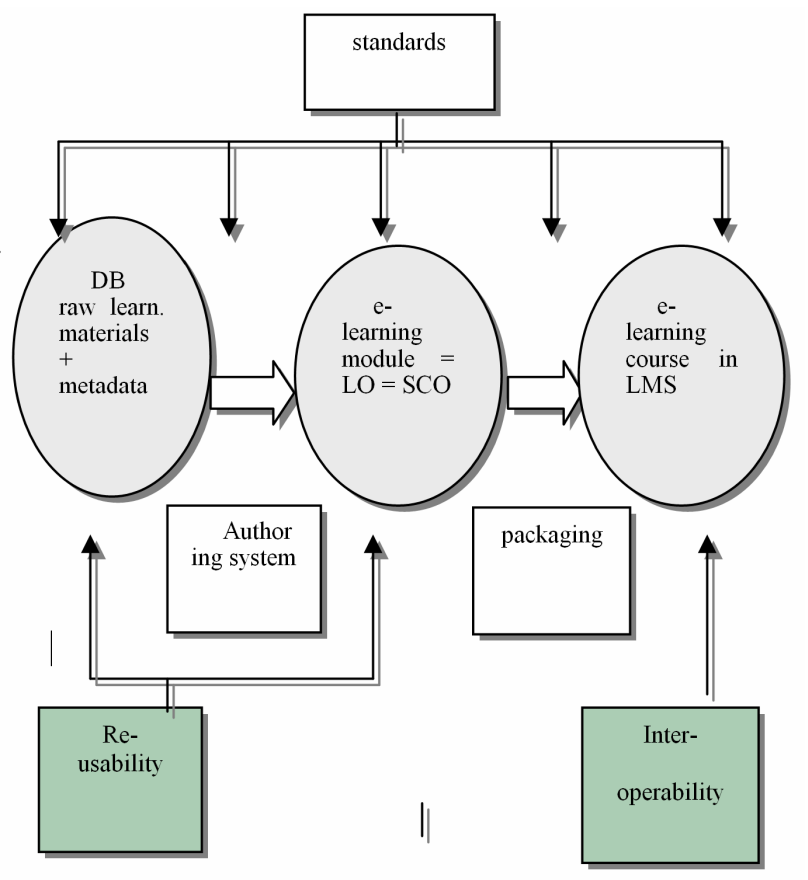

Figure 2. re-usability and inter-operability

\section{LO's customised to the preferences and the used appliance of the learner}

A dynamic LO will be created when the learner activates it. If the LO is available in more presentation layouts, then the learner can select its preferred layout.

For re-usability, we have the following possibilities in mind: first, based on the same source content, the teacher can make a selection of blocks, to bring into the LO. Second the teacher can create more LOs with the same content, but presented in another layout fitting the preference of the users and their learning styles. Third the same as the second one, but fitting the device of the user.

Different presentation templates can be developed in advance and the LO can be presented in that way.

Furthermore, mobile devices such as PDAs and mobile phones have limited presentation facilities and as a consequence in the course design the screen layout has to be simplified. Flexibility of different devices means that course materials can be automatically adjusted in order to be accessed by a variety of devices. The e-learning system will identify the type of device used by the learner and starts the e-learning course in the most appropriate built in presentation design. 
Several LO's can be built upon the same content.

The first LO includes all the source content and structured following the built in hierarchical structure. Another LO can be built on a selection of the source content. The first LO can contain the learning content on detailed level and the second one selects from all included topics the introductory blocks. F.e...

\section{Authoring SYSTEM}

\section{A. Introduction}

Authoring systems can be used to create learning content, learning modules, including text, images, speech, audio, video, ...

Some examples of commercial systems are Macromedia Authorware, Lectora of Trivantas, Toolbook of Sun Total Systems, ...

The main function of the authoring tool is the creation of learning content. Layout templates are included or can be designed by the developer. The course structure can be included and facilitates free selection of part of the course. Often facilities for creating animation and Flash objects is not included. The packages are web enabled.

Actually, authoring systems have to create re-usable SCO's. But many tools that offer SCO output are not not based on a database. As a consequence the raw content cannot be re-used and the course modules cannot be maintained or customised to the user characteristics when the user activates the course. The course has not been generated dynamically.

\section{B. Functions of an authoring system}

Once the existing content has been analysed manually and decomposed in ALO's, those ALO's have to be imported in the content database.

The database structure has to be developed taking into account the different types of ALO's and the number of content sublevels in the content tree.

The input of the ALO's can be done in two ways.

If we are working with a CMS and an authoring system which are integrated then we will import the ALO's via the input programme of the CMS. If we are working with a database based authoring system, we can use the input programme of the authoring system.

This authoring system has built in the functions of creating the tree structure, defining the titles and importing the content materials, being all ALO's. For all content components a set of metadata must be delivered too.

Once structuring and importing all materials has been finished, the result can be published, creating the SCO of this course module, or the LO of this course module content.

\section{Authoring System Creating Reusable SCO'S}

A main requirement in the development of an elearning course is the re-usability of the raw learning materials and the interoperability of the e-learning modules. We developed a quality criteria model to evaluate the authoring systems on the realization of these requirements.

\section{A. Theoretical model of quality criteria}

Based on the theory, a quality criteria model (figure(7)) has been developed. A qualitative authoring system will deliver qualitative e-learning courses fitting the requirements of re-usabilty and operability. The e-learning course will only be on the fly customisable to the characteristics of the learner who activates it, if it will be generated dynamically on time of activating it. The cost of acquiring the tool and the cost linked with the development activity is a relevant criterion too.

An instrument has been built to measure the quality of the e-learning solution on point of authoring and assembling. It is composed of a set of questions, structured around the criteria as set forward here.

- Interoperability: is the system creating SCO's or LO's which can be imported in most SCORM compliant LMS?

- Re-usability of ALO's: can the raw materials be reused to create another e-course and even by another authoring system? It means that the authoring system is working based on a database system that is not closed in the system, but is open to other authoring or user systems too.

- Maintainability of the e-course module, of the LO: if the system is based on a database, the changes of the content can easily be done in the data tables. On a dynamic way those changes will be shown in the e-learning module when being activated by the learner.

- Delivery of a customised version of the course, fitting the characteristics of the learner, being on level of learning styles and also on level of used appliances when e-learning;

- Assembling/authoring e-learning module: an easy to use user friendly solution, and delivery of attractive courses.

The tool creates the content structure and by the way the learners flexibility of selecting preferred elearning modules. The input of content and metadata is straightforward;

- Costs linked with licenses and use.

- Open authoring system: can the authoring system being customized to the organizations e-learning standards and requirements and can it be integrated in the existing system and database environment?

\section{CASE EDUWEST: AUTHORING SYSTEM CREATING REUSABLE SCO'S}

\section{A. EDUWEST and e-learning}

EduWEST.be is a Belgian non-profit organization that is offering e-learning services to educational organizations since 1999. Eduwest publishes and delivers its courses through the LCMS Digilearn ${ }^{2}$ that uses an integrated authoring tool. Digilearn ${ }^{2}$ is a product of Advanced Projects and Products (AP\&P).

\section{B. The authoring system Digilearn ${ }^{2}$}

The first version of the authoring system of Digilearn ${ }^{2}$ was created in 2004 and was aimed at authors that were not IT skilled. The first group of authors we had in mind 
were language teachers. The goal of the tool was to enable the content experts (the language teachers) to create and maintain their own content without the intervention of programmers. The experience was that intervention of programmers was very much slowing the process of content creation and maintenance.

As the LCMS Digilearn² was linked with a proprietary database from the very beginning in 1998, the integrated authoring tool is writing the content directly into the database. It's best to use a common database technology like SQL to easily transfer content to other systems and to fulfil the requirements of interoperability and re-usability of the content.

The creation of the content is made easy, although authors also can edit in the html code or add Java scripts, Flash objects, etc. The authoring tool consists of two frames: the tree structure builder is in the left frame while the editor of the content blocks, html code, pages etc. is on the right (fig(3)). In the tree structure builder the author can easily add chapters, sub-chapters and pages and can also change the structure easily. The items in the tree structure are numbered automatically by the authoring tool. You can create up to 5 levels.

The block editor offers a choice of pre-formatted templates of blocks with a different lay-out although the author still can change the lay-out. In these blocks we find the ALO's with their lay-out and the accompanying metadata. A screen in the course is consisted of one or more content blocks. The authoring tool has the ability to generate a glossary of words used in a course module.

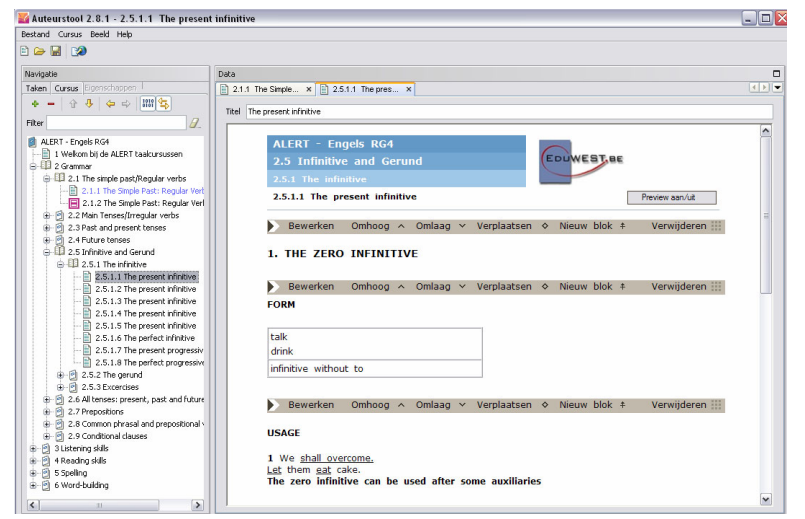

Figure 3. Digilearn ${ }^{2}$ authoring tool: building blocks

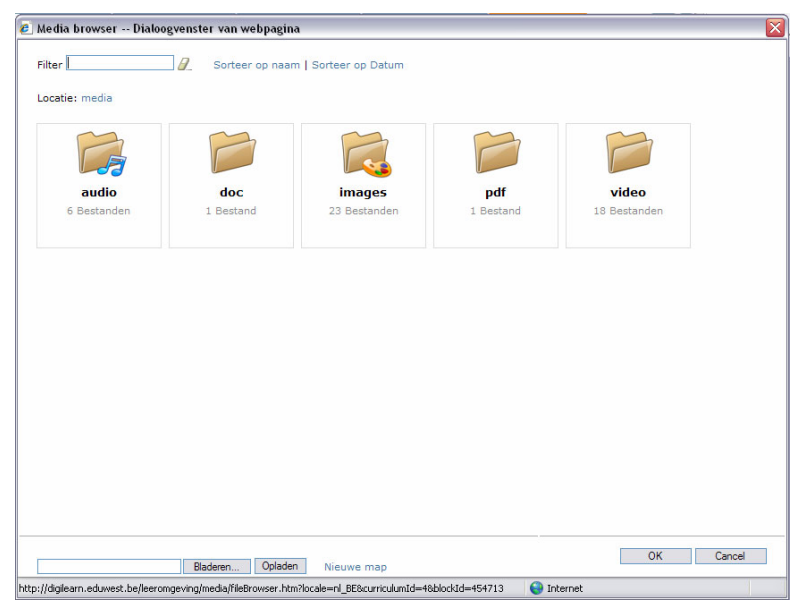

Figure 4. Digilearn ${ }^{2}$ authoring tool: media browser

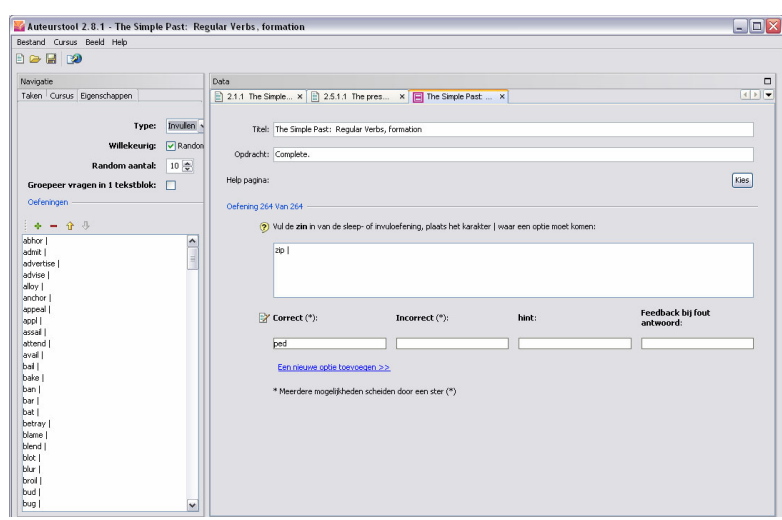

Figure 5. Digilearn ${ }^{2}$ authoring tool: exercises tool

Media files like audio, video, images, PDF/Word/Excel documents, etc. can be entered through a simple media browser with an upload functionality to easily upload the files from the local hard disk to the learning server (fig(4)).

In the exercises tool the authors can select the type of exercise (e.g. multiple choice, fill in, ...) in the left frame and add other preferences (e.g. random displaying). In the right frame the questions are edited with hint and feedback (fig(5)).

By clicking on the save button, the created content is saved into the online database of the LCMS. After publishing the content with one single click, the content is immediately available for the students in the Digilearn ${ }^{2}$ LCMS. Publishing as a SCO package makes the content available for use in other SCORM compliant learning platforms.

Working with such an authoring tool makes it possible to create individual learning paths based on the profile and the learning goals of the individual student by using the same and other building blocks.

\section{Delivery of re-usable and interoperable LO's}

We can evaluate the EduWEST.be Digilearn ${ }^{2}$ authoring tool using 7 criteria:

\section{1) Delivery of SCO's and its interoperability}

In the near future, the authoring tool used by EduWEST.be will deliver SCO's that are interoperable. Many Learning Management Systems have the functionality of packaging those SCO's. A number of metadata can be linked.

2) An easy to use solution, and delivery of attractive courses

The e-learning strategy of EduWEST.be and their educational partners is built on the active role of the expert users in the development of e-learning materials. By offering an user friendly tool that can be used by expert users with little computer skills.

The tool includes the possibility of adding learning objects of different types like Flash animations, PDF, Word, Excel, PowerPoint, video, audio, images, etc. The tool creates the tree structure of the content automatically. The content and the structure are easy to modify. It offers a quick publication of the content.

3) Low cost

EduWEST, being a non-profit organization, is distributing the Digilearn ${ }^{2}$ authoring tool and LCMS at 
low cost to non-profit educational organizations. The cost of learning to develop with the tool is very low. In 2 hours an average author can work with the tool. Of course it takes more time to master the competence of creating a good e-learning course content, but that's the same with all authoring tools. The cost is very low compared with a tailor-made programmed solution.

4) Management of portfolio of learning materials and of e-learning courses. The integration in the LCMS and the LMS.

All learning materials and metadata are organized in a database on the central server. The LCMS is a very advanced system and consists of a learning environment, an authoring tool, an administration tool, a reporting tool, an exercises tool and a built-in forum. The administration tool serves for the management of the learners, the courses, the coaches, the classes, etc. The reporting tool delivers tracking reports of learning activity and scores on exercises per learner and per class. It also delivers other management reports like a comparison of the learning activity of all the courses in the system.

\section{5) Reusability of raw materials.}

Each content block or learning object can be reused by authorized authors in the same or other course modules.

\section{6) Maintainability of the e-learning modules.}

The author can easily make changes to the e-learning modules by entering the authoring tool, making the changes, save and publish the module.

7) Dynamic generation of customised e-learning solution.

The system is based on a database to store the raw materials, but the raw materials are stored with their layout in the content blocks. For example the same raw text can be saved in a text block with some lay-out and in an audio file with the same text content in speech. So, it is not possible to create dynamic LO's where the e-learning module will be customized to the user starting the course.

ELearning systems and eLearning research areas can benefit from semantic web technologies. The Semantic Web technology has enabled by a set of suitable agents, which seems to be powerful enough to satisfy the elearning requirements fast, just-in-time and relevant learning. This allows for dynamic generation of course content according to the learner preferences, this includes:

- Distribution: using LOs that will be as decentralized as possible. This enables an effective co-operative content management.

- Personalization: A user (using personalized agent) searches for learning material customized for her/his needs. The ontology is the link between user needs and characteristics of the learning material.

- Dynamic: Enables the use of knowledge provided in various forms, by a semantical annotation of content. This distributed nature of the LOs enables continuous improvement of learning materials.

\section{The EduWEST e-learning solution is a good practice}

When the authoring tool of EduWEST will create SCO's, the e-learning modules will be interoperable. The solution is already a good practice thanks to the use of a very easy to use authoring tool and a database to store and manage the ALO's. The raw material is stored with it's lay-out, so the system cannot create dynamic solutions nor can it customize the e-learning module on the fly to the learners preference and the used appliance by the learner.

\section{CONCLUSIONS}

We set forward a dynamic generation of LO's using learning raw materials structured in a database. In view of reaching that goal, we need a LO assembling / authoring system fitting the requirements of interoperability and reusability and creating e-learning module in a dynamic way, starting from selecting the raw learning content from the learning materials content database.

The SCO's being created will be interoperable and can be imported in many Learning Management Systems.

We put together a set of quality criteria and evaluated several authoring systems based on it. The authoring systems used in practice seem not to score acceptable as compared with these criteria.

The e-learning services company developed a tool that scores very good on most of the criteria.

In the future we must evolve to systems generating the e-learning course module on a dynamic way, by generating the e-learning course module when the learner enters and that is customised to the chracteristics of the learner.

\section{REFERENCES}

[1] Jeanne Schreurs, A. Y. Al-Zoubi: A blended Learning Concept for guided self-instruction. Proceedings of IMCL2007 Amman; ISBN 978-3-89958-276-5.

[2] Jeanne Schreurs, G. Ballet, R. Moreau, “An E-learning Concept Mindmap and the Management of the Underlying Learning Objects", Lecture Notes in Computer Science. Publisher: Springer-Verlag Heidelberg ISSN: 0302-9743, Vol. 3292/2004 : Title: On the Move to Meaningful Internet Systems 2004. Editors: Robert Meersman, Zahir Tari, Angelo Corsaro; ISBN: 3-540-23664-3 DOI: 10.1007/b102133, p. 758.

[3] J. Schreurs, R. Moreau: "Learning Objects Aligning Different Learning Styles", Proceedings of the nternational Conference on e-Learning, ICEL2006; University of Quebec Montreal, 22-23 June 2006, pp 415-422, ISBN 1-905305-31-5. (doi:10.1076/ilee.9.3.219.3573)

[4] N. Friesen (2001) "What are Educational Objects?", Interactive Learning Environments, 9:3, p. 219-230.

[5] Wiley, D. A. (2000). Connecting Learning Objects to Instructional Design Theory: A Definition, a Metaphor, and a Taxonomy. In D. A. Wiley, The Instructional Use of Learning Objects. Bloomington: Association for Instructional Technology en Association for Educational Communications and Technology.

[6] I. Varlamis and I. Apostolakis, (2006), The Present and Future of Standards for E-Learning Technologies. (A. Koohang, Red.) Interdisciplinary Journal of Knowledge and Learning Objects, 2, 59-76.

[7] Masie. (2002, maart 8). Making Sense of Learning Specifications and Standards: A Decision Maker's Guide to their Adoption. Opgeroepen op mei 28, 2007, van The MASIE Center: http://www.masie.com/standards/S3_Guide.pdf.

[8] K. Ryann: "E-Learning Standards Update", Learning Circuits 2005 http://www.learningcircuits.org/2005/jul2005/ellis.htm.

[9] J. Schreurs, R. Moreau: "Converting Learning Content to Learning Objects (LO) and Atomic LO's", Proceedings of the Virtual Conference: IADIS Virtual Multi Conference on Computer Science and Information Systems, MCCSIS 2006, 15-189 May 2006, ISBN 972-8924-14-3.

[10] A. Strijker: "Het maken van leerobjecten binnen de Digitale Universiteit" Digitale Universiteit 2006. http://www.surffoun dation.nl/du/MakenLeerobjecteDUhandboek.pdf. 
[11] C. Larman, “(2002) Applying UML and Patterns”, United States of America, Prentice-Hall.

[12] Shepherd, C. (2000). Objects of interest. Opgeroepen op mei 28, 2007, van Fastrak Consulting Ltd.http://www.fastrakconsulting.co.uk/tactix/features/objects/objects.htm

[13] http://lspserver.uhasselt.be/learning/Loms1280.htm

[14] http://lspserver.uhasselt.be/eschemapers/mindmap/supply_of_ict_ professionals.asp

[15] J. Schreurs, A.Y. Al-Zoubi: "Converting Content to Reusable Learning Objects Adaptable to User Preferences and Infrastructure"; Proceedings ELEARN2007; Quebec; ISBN: 1880094-63-0.

[16] Harman, Keith en Koohang, Alex. 2007a. Learning Objects: Applications, Implications, \& Future Directions. Santa Rosa : Informing Science Press, 2007.

\section{AUTHORS}

J. Schreurs (Jeanne.Schreurs@uhasselt.be) is Professor at the Faculteit Toegepaste Economische Wetenschappen, Universiteit Hasselt, Campus Diepenbeek, Agoralaan gebouw D, 3590 Diepenbeek, Belgium.

Rik Dalle (rik.dalle@eduwest.be) is coordinator of EduWEST.be vzw, Korte Meersstraat 6, 8900 Ypres, Belgium.

George Sammour (george.sammour@uhasselt.be) is a $\mathrm{PhD}$ student at the Transportation Research Institute, Hasselt University, Diepenbeek, Agoralaan gebouw D, 3590 Diepenbeek, Belgium.

This article was modified from a presentation at the IMCL2009 conference in Amman, Jordan, April 2009. Manuscript received 26 January 2009. Published as submitted by the authors. 